(C)2009 IEEE. Personal use of this material is permitted. However, permission to reprint/republish this material for advertising or promotional purposes or for creating new collective works for resale or redistribution to servers or lists, or to reuse any copyrighted component of this work in other works must be obtained from the IEEE. 


\section{Remote Laboratory Access for Students with Vision Impairment}

\author{
Iain Murray \\ Department of Electrical \& Computer Engineering \\ Curtin University of Technology \\ Perth Australia \\ i.murray@curtin.edu.au
}

\author{
Helen Armstrong \\ School of Information Systems \\ Curtin University of Technology \\ Perth Australia \\ h.armstrong@curtin.edu.au
}

\begin{abstract}
The delivery of laboratory exercises to students that are unable to attend in person due to physical disabilities is a significant issue. Both Netlab and Packet Tracer are inaccessible to many students who use assistive technology, particularly those with vision impairment. This paper presents the development of an accessible, cost effective, remote laboratory and describes the modification to laboratory sessions necessary for the blind to undertake CCNA laboratory sessions remotely and with full accessibility. Also discussed is the development of an accessible network simulator, iNetSim, illustrating possible methodologies that may be applied to make existing simulation packages accessible to those with severe vision impairment.
\end{abstract}

Keywords-component; Networking laboratories, vision impaired, accessible eLearning

\section{INTRODUCTION}

Curtin University of Technology commenced offering the Cisco Network Academy Program to mainstream (sighted) students as part of the Bachelor of Technology (Computer Systems \& Networking) degree program in 2002. Shortly after, four vision impaired students expressed an interest in entering the Bachelor of Technology program. These students faced significant problems with accessibility to the Cisco course on-line materials as much of these materials were not accessible to non-sighted users. Extending the Cisco courses to vision impaired students posed numerous teaching and learning challenges.

The Cisco Academy for the Vision Impaired (CAVI) has been delivering the Cisco Academy Programs to blind and vision impaired students since 2003, with up to 9 students per year from the local area. In 2007 the program was expanded to include students located in other parts of Australia and the U.S.A, with 25 vision impaired students enrolled in that year. In 2008 the enrolments of legally blind students exceeded 120, from countries including India, Sri Lanka, Canada, Egypt, Australia and the U.S.A. During the intervening period, a number of vision impaired students entered the Bachelor of Technology (Computer Systems \& Networking) course at Curtin University with the total number of vision impaired students (in the CAVI program) increasing to 146 in the year 2008. In order to achieve the practical components of the CCNA courses, remote access to router and switch bundles was required. This paper describes the remote laboratory established for these students together with a network simulation application developed.

\section{A. The Mechanics of Delivering the Curricula}

The Cisco curriculum is "media rich", with much of the content delivered as Flash and interactive web pages. This style of delivery is often unsuitable for vision impaired persons. The arrangement of frames is inaccessible to screen review applications (speech output), but more importantly the curriculum relies heavily on visual keys to illustrate learning objectives. Several problems, not apparent to most sighted users, are also inherent in the curriculum design. The first problem is that the diagrams are extremely difficult to access or even explain to a person who has been blind since birth. The second problem is that the arrangement of frames and the lack of correct ALT labels (text equivalent buttons) add to the complexity of the presented material. The vision impaired students also advise they have no way of accessing the content of interactive sessions and find the supporting text confusing and misleading. To overcome these issues many supporting applications and documents were created but are beyond the scope of this document.

The CAVI program utilizes blind instructors to deliver the Cisco course materials with the support of a sighted teaching assistant. Blind instructors have first hand experience of the difficulties encountered by the vision impaired students and understand the most effective ways of presenting the materials. The classroom environment consists of a laboratory containing a network of PCs fitted with assistive technologies, routers and associated network equipment. Classes run two full days per week over the academic year. Local students physically attend classes and 
remote students $\log$ in (via the Internet) to a virtual classroom to listen to the lectures and participate in the tutorial exercises. The virtual classroom provides the facilities for students to talk to one another as well as communicate with the instructors, similar to a normal classroom environment. The lectures are recorded and made available as audio files on the project website along with other teaching materials for access by the students at any time.

It may be argued that the most difficult issue in delivering eLearning to blind students is that of explaining the meaning of graphical information. To overcome this issue, textual descriptions were created for all graphics used in the courses, including the curriculum, laboratory manuals and on-line exams. An example textual description is given in the excerpt below and refers to the diagram in Figure 1, graphic 2 of 4 in the text description.

\section{Page has 4 graphics}

Graphic 1 shows the segmentation with routers. There are four hubs and one router in the picture. Router is in the middle connected to hubs in four corners, three stations (PC) are connected to each hub.

Segmentation with routers provides:

More manageable, greater functionality, multiple activate paths

Smaller broadcast domain

Operates at layer 3

Graphic 2 show routers connected by WAN technologies. There are 10 routers in the graphic. Four of them are connected to each other in a square shape (each one corner of a square). If we name these routers from 1 to 4 clockwise starting from the left top, router number 1 is connected to router number 2 with ATM (Asynchronous Transfer Mode). Router number 2 is connected to router number 3 with T1/E1 and T3/E3. Router number 3 is connected to router number 4 with ATM. Router number 4 is connected to router number 1 with T1/E1 and T3/E3.Each of these four routers are connected to other routers.

Router number 1 is connected to two other networks one a cable modem via a router and other one X.25 via other router.

Router number 2 is connected to a dial-up modem via a router.

Router number 3 is connected to SDMS via a router and to $x D S L$ via another router.

Router number 4 is connected to ISDN network via a router.

There are antenna signal to router number 3 and satellite signal to ISDN router connected to router number 4.

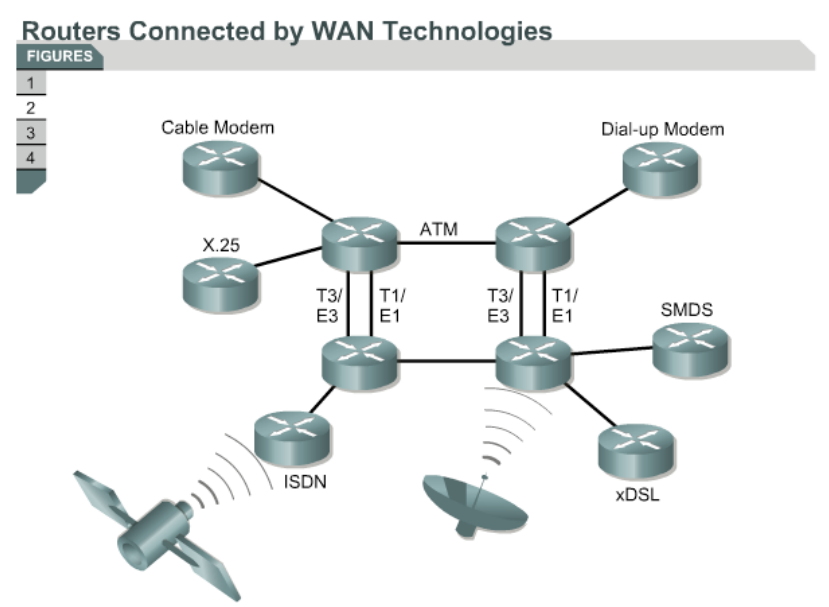

Figure 1: Example diagram from CCNA2 version 3.1 section 1.1.3

Further details of the teaching aids used in the project can be found in Murray and Armstrong [3] and on the project website http://www.cucat.org.

\section{THE REMOTE LABORATORY USER EXPERIENCE}

Laboratory exercises form a significant portion of the curriculum. In order for students situated remotely to access and participate in the laboratory sessions, a functional, remotely accessible network topology was developed. The configuration illustrated was developed for the CCNA version 3.1 curriculum, with work currently underway to reconfigure for Discovery and Exploration curricula to be delivered in 2009. Laboratory equipment generally consists of three routers and two switches. The configuration may be described as two branch offices, say Perth and Sydney, connected together via the ISP or Internet cloud [1]. The edge routers are configured by the students to allow connectivity via the middle router (cloud or ISP). Local students interact directly with the routers' configuration via serial (console) interfaces. A problem exists when attempting to allow remote students access to "real" routing hardware. The routers may not be placed on production networks for obvious reasons and initial configurations must be entered via the console connection. Therefore requirements for a remote lab must allow students to perform:

- Initial configuration via the console cable

- Remote power cycling of network equipment and workstations

- Connectivity tests

- Advanced router and switch configuration.

One such system does exist, Netlab, developed and distributed by NDG (http:/www.netdevgroup.com/), however the cost of this system is a major factor hindering its adoption. Additionally, the java based applications in Netlab, including the booking system, telnet client to interact with the network hardware and server system are not 
accessible by screen readers (software utilized by blind computer users to convert on-screen information to audio or Braille output). The CAVI system developed costs significantly less than the Academy Edition of Netlab. Whilst it does not offer advanced features such as equipment booking it performs all the required functions for the vision impaired class applications. In its most simple form, it consists of the standard CCNA laboratory bundle: 3 routers and 2 switches, with several virtualized Linux PC servers running FTP, HTTP, Telnet and other associated services; all may be accessed by their serial ports (see Figure 2).

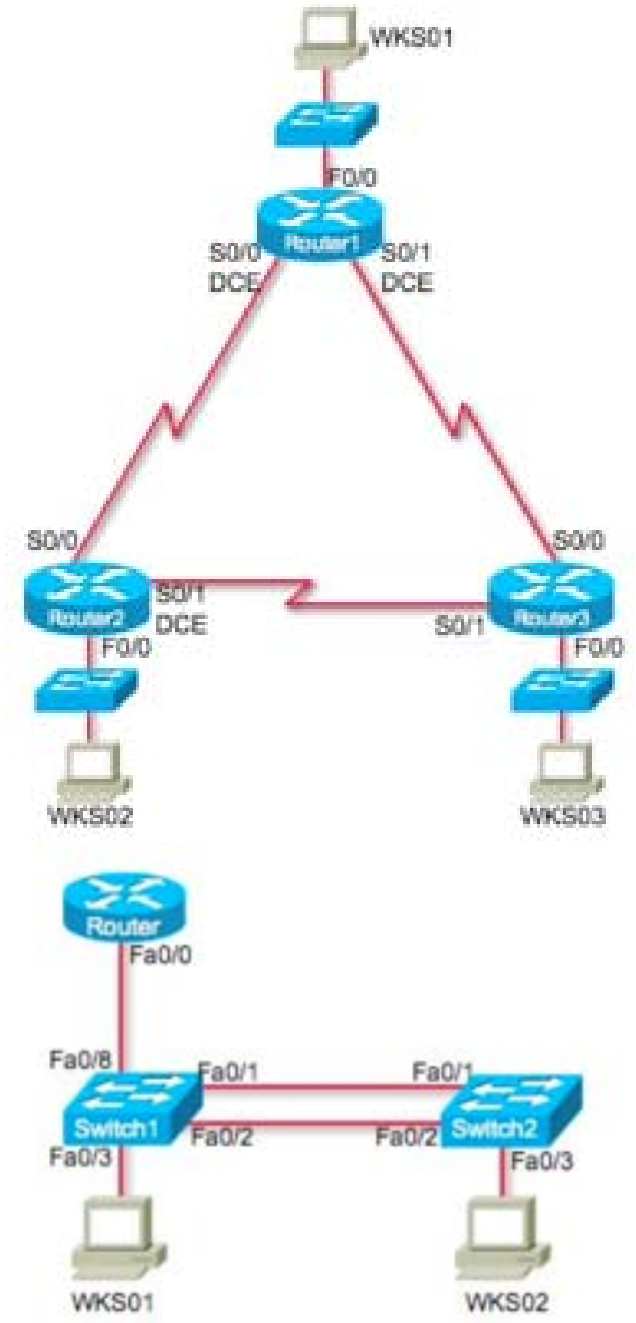

Figure 2: Standard router bundle (top) \& Switch Bundle (bottom)

A standard serial port or console switch is used to access the devices in the laboratory bundle. Remote students may telnet into the console server (a device that allows Ethernet to multiple serial port connections), accessing the routers, switches and Linux servers from any locality worldwide. The use of Linux on the host and server machines is necessary as the command line may be accessed through the serial ports allowing the students to connect directly to the server hosting the multiple virtual machines. Users may then telnet to the virtual machines and access the command line via the screen reader. Virtualized GUI based operating systems are not easily accessible to the assistive technology when installed behind the console switch.

The physical layout of the remote laboratory equipment is depicted in Figure 3.
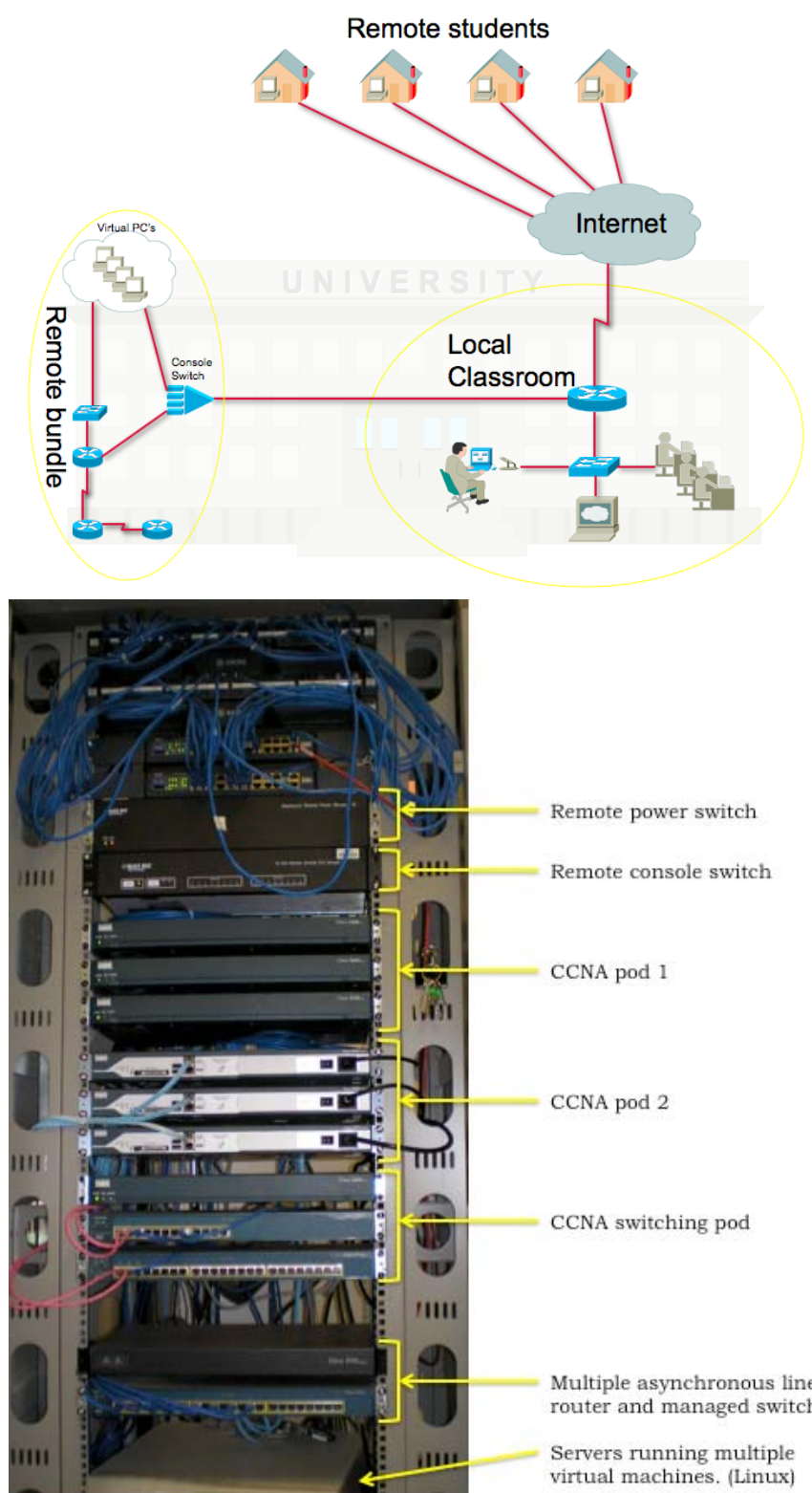

Figure 3: Teaching environment (top) and physical remote laboratory equipment layout (bottom)

Figure 4 illustrates an active telnet session logged into the remote bundle. As the routers are on their own network, with remote access attaching only to the serial ports, this system does not offer any security risk to the institution utilising it. Once the student has authenticated with the console switch (simple plain text password) a list of available equipment is displayed, as shown in Figure 5. 


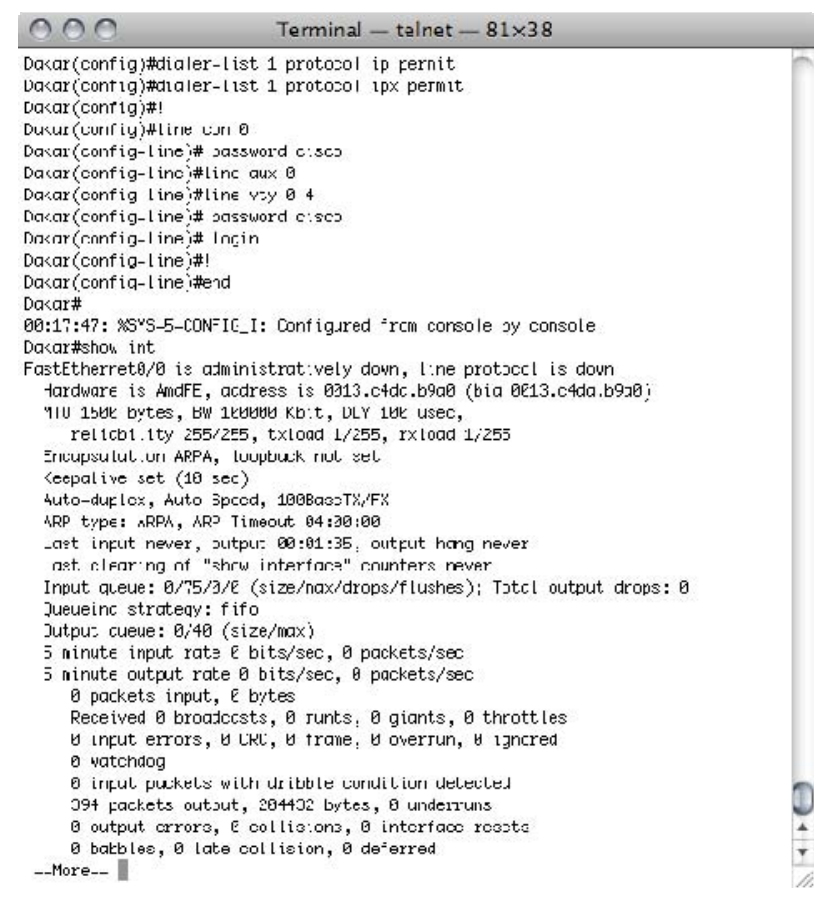

Figure 4: Active console session

[Murray-2: ] iainmurrayo telnet 134.7.43.171

Trying 134.7.43.171...

Connected to 134.7.43.171.

Escape character is '^ $]$ '.

Enter Password: $* * * * * * *$

PORT STATUS: Version 3.0, Site ID: Curtin -o o- --oo o- -o oo o

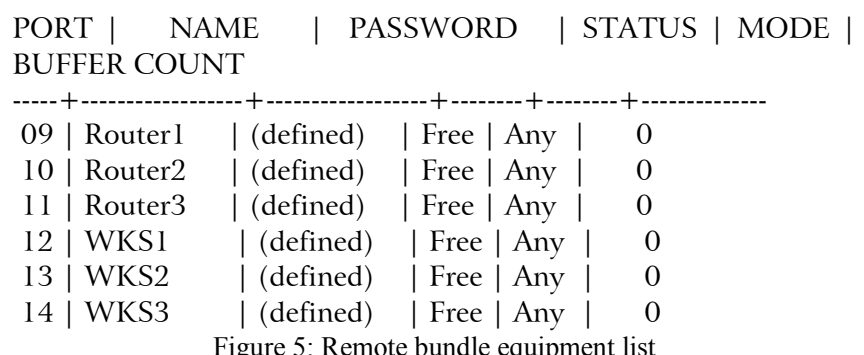

Several commands are available and are listed in Table 1. Connection to equipment is made via the / $\mathrm{C} \mathrm{n}$ command, where $\mathrm{n}=$ required equipment port number.

TABLE I. TABLE 1: CONSOLE SWITCH COMMAND MENU

\begin{tabular}{|l|l|}
\hline Display Options & \\
\hline$/ \mathrm{S} / \mathrm{SD}$ & Port Status \\
\hline$/ \mathrm{W}$ & Port Parameters (who) \\
\hline$/ \mathrm{J}$ & Site ID \\
\hline$/ \mathrm{H}$ & Command Menu (Help) \\
\hline Control & \\
\hline$<$ Enter $>$ & Enter Command Mode \\
\hline$/ \mathrm{x}$ & Exit Command Mode \\
\hline$/ \mathrm{Cn}$ & Connect to Port (n: Port\# or name) \\
\hline
\end{tabular}

As the booking system was incomplete at the time of writing, a virtual classroom was utilised as a method of ensuring students knew if the equipment was in use. When undertaking a laboratory, students logged into the Ventrilo server (a voice communication application designed for online gamers) and entered the appropriate channel, as shown in Figure 6. In this way students not only can tell if a particular bundle is in use but may also conduct laboratory sessions collaboratively with other students.

Power cycling of equipment is undertaken by authenticating first to the console switch and connecting to the remote power switch. The power switch may then be used to power down individual devices within the bundle. This is usually done with a secondary telnet session, allowing access to the router/switch to be power cycled and therefore the boot process to be interrupted (as in the case of password recovery laboratories). Each device may be powered on, off or rebooted. Figure 7 illustrates the process of remotely rebooting a router.

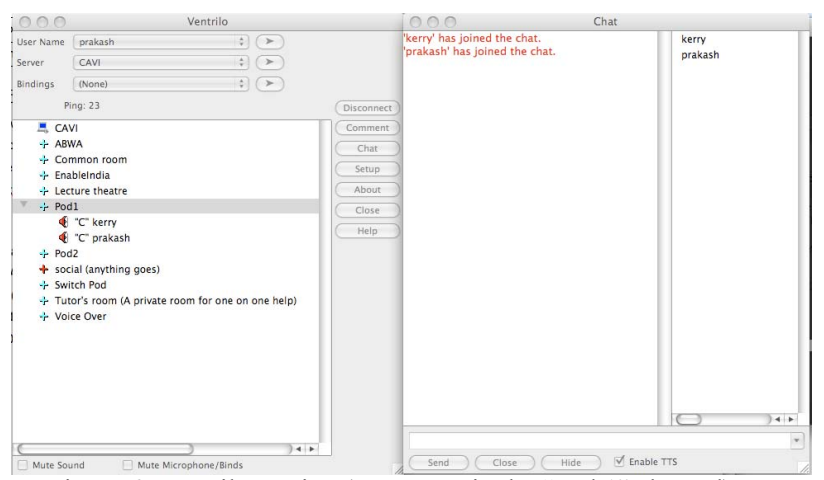

Figure 6: Ventrilo session (note users in the "Pod 1" channel)

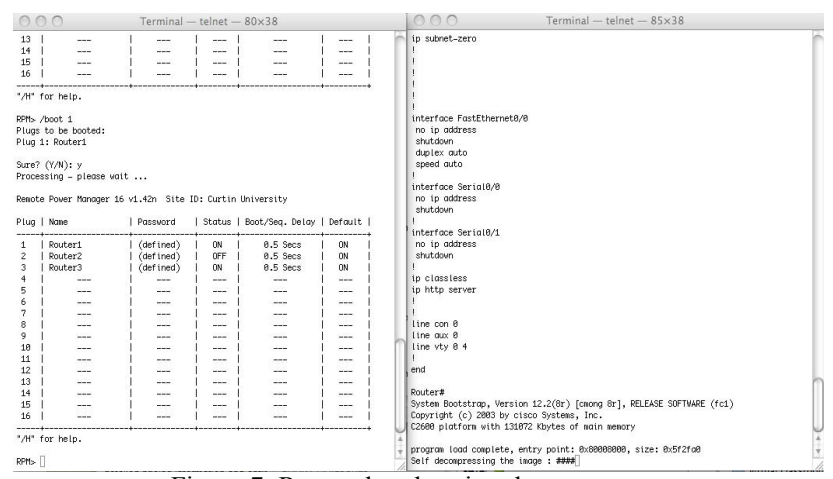

Figure 7: Remotely rebooting the router

Once connected via telnet, students may configure routers, switches and workstations in the same manner as if physically present. One such session is shown in Figure 7. Although this system allows laboratories to be completed, including e-Labs it has several shortcomings. These include the lack of a formal booking system, leading to students attempting to access the limited resources while others are 
engaged in laboratory sessions and it does not give students experience with the physical cabling of network systems. To assist students with understanding the physical aspects of cabling, recorded audio demonstrations of the physical features of cables, connectors and their locations on switch and router hardware are conducted. In these demonstrations, a local vision impaired student conducts a supervised cable lab, describing in detail what they "feel" and how connections are made, in much the same manner as video is utilized for sighted students.

\section{INETSIM NETWORK SIMULATOR FOR APPLE OS X}

iNetSim is a accessible network simulator, created to allow both vision-impaired and sighted users to complete CCNA 2 laboratory sessions without access to the networking hardware [2]. Existing software used in the CCNA course for network simulation and laboratory practice (Packet Tracer) and the eLabs is not accessible to those with impaired vision as it utilises images of network topology, allows only mouse selection of network devices and tools and is incompatible with screen reading software. In contrast, iNetSim has been developed to be accessible by blind and vision impaired users in addition to those with normal vision. All user interface and network topology elements are accessible via the Apple screen reader (VoiceOver) keyboard shortcuts and provide a meaningful response when read by VoiceOver. Network simulators usually rely on the use of a mouse to add simulated communication links between devices, place network devices on the work area canvas, select configuration options and view simulation results. To connect two devices with a communications link, the user must generally click on icons for the simulated devices and drag the connection to its end point, another network device under normal circumstances. As this is usually not possible for visionimpaired users, iNetSim also incorporates the use of tables for connecting devices. Tables are used to alter a device's location in the topology area, and configure ports and links. Tables are used as navigation with speech prompts, can be achieved with VoiceOver shortcut keys and cursor keys. iNetSim can be used solely with the keyboard, therefore the eye and hand issues faced by vision-impaired students can be avoided. As a GUI is also available, sighted iNetSim users can alternatively use a more traditional drag-drop mouse-based interface.

The system is capable of representing several generic network devices including routers, switches, hubs and PCs. Each device must be configured via a command line for correct operation. Figure 8 illustrates the application running with the textual command line terminal session to Router0 open. Note that the IP address on Router0, interface $\mathrm{S} 1$ is set to the same value as in the port table (highlighted) under the main canvas. Selecting values from the drop down boxes or edit fields in the main application window has the same effect as entering the command line configuration. Changes made with either method will be reflected throughout the application. This allows rapid basic configuration to be undertaken by the instructor so that students may concentrate on the particular task in the session.

Each device may have several ports of different types including Ethernet, serial and console. The user creates a connection by specifying two ports to connect and a cable type. Removing a connected port disables the connection the same way unplugging a cable would in a real network. The command line interface to devices also provides control and feedback over the simulation. The interface acts in a similar way to the operating system for that device type (e.g. a generic DOS-like system for PCs and Cisco IOS for routers). A subset of the commands applicable to CCNA 2 allows the user to display and modify device configuration, establish routing protocols and ping, Traceroute or telnet to other devices. iNetSim maintains a representation of routing tables to simulate these tasks correctly.

A completed laboratory is depicted in the screen capture illustrated in Figure 8. Note the configuration entered in the terminal screen matches the configuration in the tables and the successful pings from both the routers and workstations.

\section{PACKET TRACER EXERCISES AND ELABS}

The combination of access to real network hardware and a practice environment in the simulation software has shown to be a valuable resource. CAVI has converted Discovery Packet Tracer exercises and eLabs (from CCNA version 3.1 ) to a format that may be run on the remote bundles so that students receive a similar learning experience to that of their sighted peers. These conversions include descriptions of graphics and network topologies, starting router configurations that are pasted into the routers and accessible instructions on laboratory procedure. The disadvantage is in cases where a large number of network devices are required, simplified topologies were used due to restrictions on the quantity of network devices in the remote bundle. 


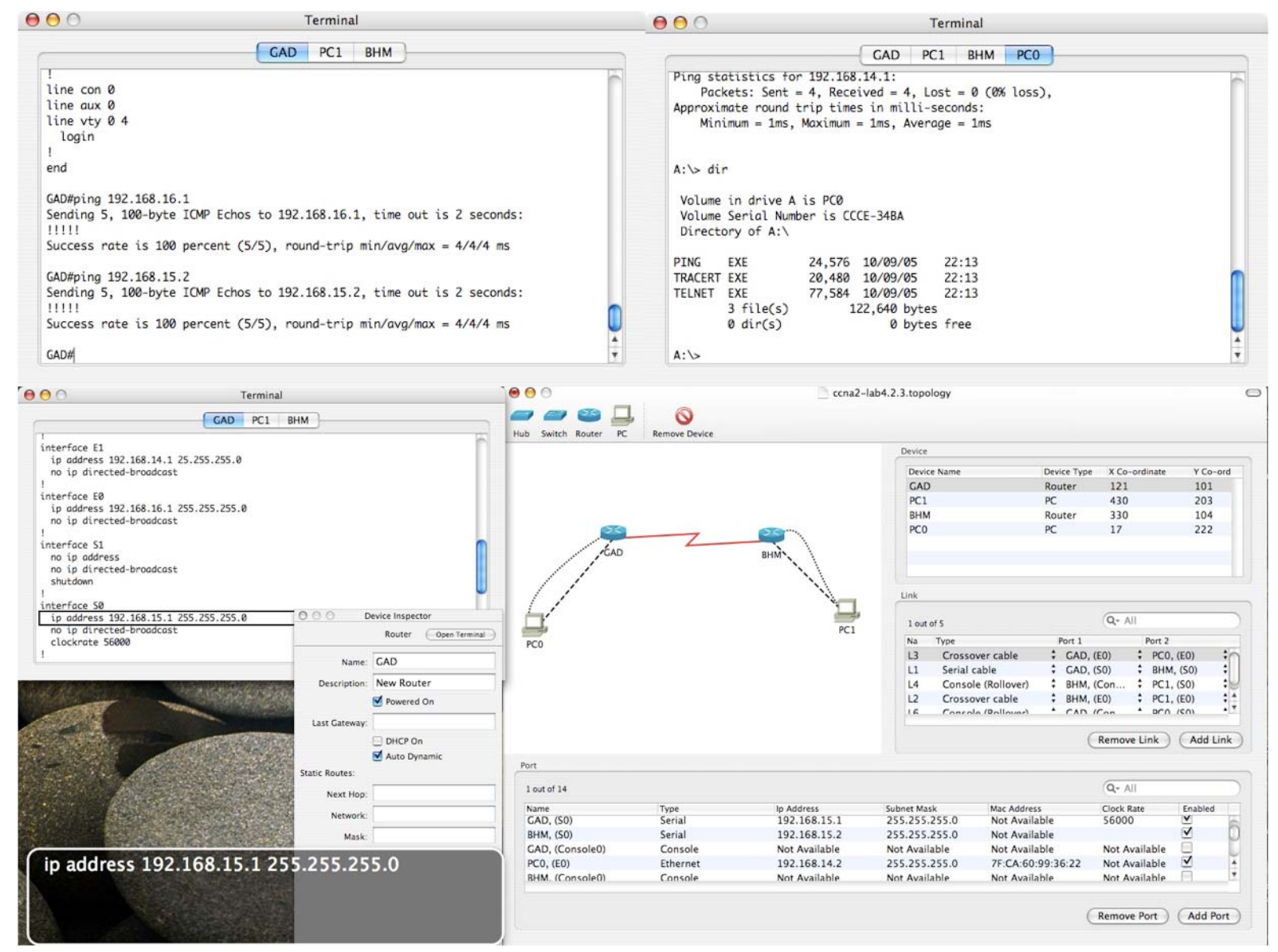

Figure 8: iNetSim with command line terminal session open. The text in the floating window shows the text read by VoiceOver

\section{CONCLUSIONS}

Whilst the use of the remote bundle overcomes many of the limitations imposed by delivering CCNA laboratories to remote blind and vision impaired students, further work is required to improve functionality and ease of use. A method of booking and authentication of users is currently under development as part of a "front end" web portal to streamline the connection to network devices.

iNetsim was successful in proving that network simulators may be made accessible. However, it is now considered that, with the availability of Packet Tracer API's and the Packet Tracer Messaging Protocol a possible way forward in accessibility is to develop an accessible extension (a user interface) that communicates with Packet Tracer. This would have the benefit of utilizing the superior Packet Tracer library of devices and protocols and the support and continuing development without duplication of resources.

The two solutions presented in this paper assist in overcoming the laboratory issues involved in remote delivery to vision impaired students however there are many significant obstacles in accessibility that have also been addressed in the CAVI project [3]. The CAVI classes offer a holistic environment tailored to cater for blind and low vision students without compromising course quality and student outcomes. Using the environment and tools established by CAVI students with severe vision impairment are able to undertake the same Cisco courses as their sighted counterparts.

\section{REFERENCES}

[1] H. Armstrong and I. Murray, "Remote and Local Delivery of Cisco Education for the Vision-Impaired", ITiCSE 2007, Dundee Scotland, 25-27 June 2007, pp78-81

[2] J. Hope, B. vonKonsky, I. Murray, L. C. Chew and B. Farrugia, 'A Cisco Education Tool Accessible to the Vision Impaired', ASSETS06, Portland, Oregon USA, October 23-25, 2006, pp235236

[3] I. Murray and H. Armstrong, "Innovations in tertiary online learning for students who are blind or vision impaired", $7^{\text {th }}$ International Conference on Information Technology Based Higher Education and Training, Sydney Australia 10-13 July 2006 